\title{
Deconvolution of IR Spectra by Functionally-Enhanced Derivative Spectroscopy (FEDS): Why Does It Work?
}

\author{
Manuel Palencia ${ }^{1}$ \\ ${ }^{1}$ GI-CAT, Department of Chemistry, Universidad del Valle, Cali-Colombia
}

Corresponding Author: M. Palencia. E-mail: manuel.palencia@correounivalle.edu.co

Graphical Abstract
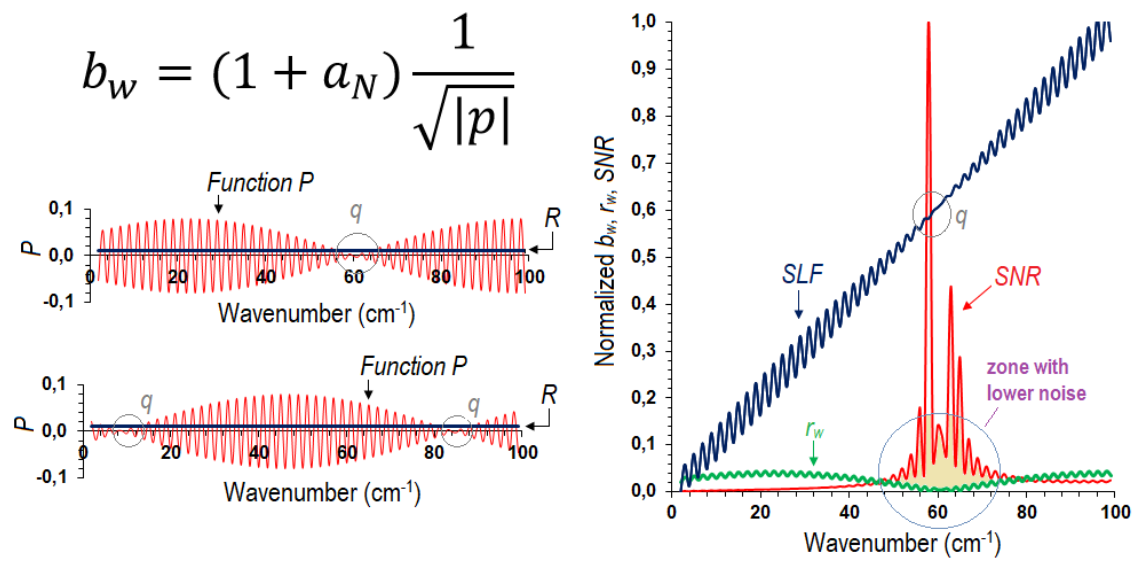

\begin{abstract}
Functionally-enhanced derivative spectroscopy (FEDS) have been proposed as a tool for the easy spectrum deconvolution by the analysis of spectrum line function. However, given that FEDS was deduced from end-spectrum mathematical transformations using simple mathematical operations, some aspects to explain how FEDS can detect signals and differentiate them from non-signals are not completely understood. In order to increase the understanding of FEDS transform and expand its use in the analysis of HCM-systems, the residual noise remaining in the working mid-infrared spectra was studied and connected with analysis by FEDS. To exemplify the concepts developed, mid-infrared spectra of Escherichia coli were used as model of HCM-systems. It was demonstrated that endspectrum noise is related with standard deviation of signal fluctuation in the spectrum permitting the easy calculation of signal-noise ratio (SNR) and the obtaining of SNR as a function of wavenumber from working spectra. In addition, our results demonstrate that spectrum obtained by FEDS transform is mainly a SNR-based spectrum, being a result of FEDS algorithm to find the data with maximum SNR and separate them by the use of simple conditional functions. It is concluded that spectral deconvolution can be performed easily by FEDS, permitting the separation of overlapped signals and the detection of hidden signals with high-SNR, with capacity to be generalized for numerous types of spectra.
\end{abstract}

Keywords: Signal-noise ratio, derivative spectroscopy, data transformation, biolayer, infrared

Cite as: Palencia M. Deconvolution of IR Spectra by Functionally-Enhanced Derivative Spectroscopy (FEDS): Why Does It Work?. J. Sci. Technol. Appl. 9 (2020) $18-28$. 


\section{Introduction}

When an analytical measurement is performed, two basic components can be identified: A first one component is the signal that is understood as the fraction of information associated with the property of sample which are being investigated and, the second one is the noise that is undesirable information that affects the accuracy and precision of analysis, the detection limit and, in some cases, impossibilities the quantitative determination of analyte [1-2]. Particularly, into spectroscopic techniques, the effect of noise is very important when highly-complex multicomponent samples (HCMSs) are being analyzed, such as, biofilms, soils, plant extracts, tissue and biological fluids [3-4]. The main descriptor for the monitoring of effect of noise in spectroscopic analysis is the signalto-noise ratio (SNR), which compares the amount of useful information (signal) with the noise in the analytical response. Usually, SNR can be defined as the ratio between the signal mean and the corresponding standard deviation (i.e., $\mathrm{SNR}=\mu / \sigma$ ) for a set of spectral replicas [2].

The use of SNR as descriptor of quality of analytical method, in most measurements, assumes that the average power of the noise is constant and independent of the magnitude of the signal [1-2, 5]. However, Howard and Griffiths (2002) identified that, in techniques as mid-infrared or ultraviolet-visible spectroscopies, the noise in the spectrum is not constant unlike it established by noise classic derivation in spectroscopy [6]; but also, our research team during analysis of HCMSs have identified the presence of non-constant noise distribution along the spectrum which is usually neglected during the analysis. In fact, the above observation is not new, in many cases, in the most of qualitative spectroscopic analysis for pure, low-complexity or few-component samples the effect of noise contained in the end-spectrum is neglected and assumed to be a constant (here, end-spectrum noise is defined to be the residual noise that is not eliminated during the collection and processing of data and, therefore, it is transmitted to the working spectrum resulting of spectroscopic analysis). However, the effect of this endspectrum noise on the spectrum analysis is significant in quantitative applications, such as, determination of thin film thickness by the analysis of penetration deep using Fourier transform infrared by attenuated total reflectance (FTIR-ATR) [7]; or when digital techniques as Fourier self-deconvolution [8-9], derivative spectroscopy [10-11], and functional-enhanced derivative spectroscopy (FEDS) [12] are used, including convolutional neural networks for multivariate regression [13] and principal component analysis [14] among other.

On the other hand, the use of single spectrum for the analysis of endspectrum noise has been previously proposed as a simple test for instrumental quality control in laboratories [15]. By this proposal, the extraction of noise is carried out by two steps (i) the smoothing of spectrum using the Savitzky-Golay algorithm and (ii) the smoothed spectrum is subtracted of the original spectrum. Thus, the prediction of "real" spectrum is carried out from the working spectrum and the resulting changes are related directly with the noise; in addition, the main reason for the use of Savitzky-Golay algorithm is that this is widely generalized and, in consequence, in a strict sense, the estimation of "real" spectrum could be performed by any smoothing algorithms [16-17]. Recently, Bazar et al (2016) applied the above ideas for the evaluating of analytical signals in order to identify the spectral error identifying the presence of systematic error in the working spectra during routinely quantitative analysis [18-19].

Here, the concept of end-spectrum noise for spectroscopic techniques by the analysis of spectrum line function (SLF) from geometric and statistical arguments is developed in order to be used in the enhancing of the spectral analysis. In addition, the connection of end-spectrum noise with the SNR and its application in the spectrum digital processing by FEDS are discussed. Finally, concepts developed are exemplified in the analysis of mid-infrared spectrum of $E$. coli biolayers, however, these could be generalized for other type of spectra and samples.

\section{Theory}

\subsection{Geometrical and statistical descriptions of end-spectrum noise}

The spectrum-contained noise $(R)$ can be defined to be the average of noise $(r)$ in function of the wavenumber $(w)$ and it is the fluctuation of SLF around a central value. Here, $r$ is geometrically defined to be the average distance between two consecutive values of absorbance $\left(b_{w}\right)$ throughout spectrum segment that is being analyzed. Thus, $R$ is given by

$$
R=\frac{1}{n-1} \sum r_{w}=\frac{1}{2(n-1)} \sum\left|b_{w+1}-b_{w}\right|
$$

where $n$ is the number of points in the analysis window. Given that $\Delta w=w_{j+1}-w_{j}, n$ can be calculated by

$$
n=\frac{\left(w_{\max }-w_{\min }\right)}{\Delta w}
$$

where $w_{\max }$ and $w_{\min }$ are the maximum and minimum values of $w$ in the analysis window, respectively. Though $R$ can be used to define a base line from of information self-contained in the spectrum, $r$ is seen to be useful parameter for the analysis of signals in a specific spectral region.

From statistical point of view, $R$ can be understood as the average standard deviation $\left(s_{m}\right)$ calculated from mean variance between pairs of consecutive data. For this, the average between $b_{w}$ and $b_{w+1}$ $\left(b_{m}\right)$ is assumed as a better estimation of the signal corresponding to $w+1$. Thus,

$$
b_{m}=\frac{b_{w+1}+b_{w}}{z}
$$

where $z=2$ (here, $z$ is used to obtain a generalized expression congruent with Savitzky-Golay algorithm). On the other hand, sample variance $\left(s^{2}\right)$ is 


$$
s^{2}=\frac{\left(b_{w+1}-b_{m}\right)^{2}}{z-1}
$$

In the equation 4 , because sample average for one pair of consecutive data in the SLF is unknown, the Bessel correction was applied. Combining the equations 3 and 4 is concluded that

$$
s^{2}=\frac{\left[(z-1) b_{w+1}-b_{w}\right]^{2}}{z^{2}(z-1)}
$$

By the above, the standard deviation $(s)$ is given by

$$
\begin{aligned}
& s=\sqrt{\frac{\left[(z-1) b_{w+1}-b_{w}\right]^{2}}{z^{2}(z-1)}} \\
& s=\frac{\left|(z-1) b_{w+1}-b_{w}\right|}{z \sqrt{z-1}}
\end{aligned}
$$

For pairs of consecutive data $z=2$ along the $n-1$ consecutive data, $s_{m}$ is

$$
s_{m}=\frac{1}{n-1} \sum \frac{\left|b_{w+1}-b_{w}\right|}{2}
$$

Finally, from equations 1 and 8 , it is concluded that $R=s_{m}$, but also, $r$ is completely congruent with geometrical description implicitly defined in the equation 1 , i.e.,

$$
r=\frac{\left|b_{w+1}-b_{w}\right|}{2}
$$

In a generalized form, as a function of $w$, equation 9 can be rewritten to be

$$
r_{w}=\frac{\left|b_{w}-b_{w-1}\right|}{2}
$$

\subsection{Functional-enhanced derivative spectroscopy (FEDS)}

FEDS transform is an algorithm for digital analysis of spectra based on functional transformation of SLF; thus, spectral transformation is performed using mathematical and logical functions which are defined from original data [12]. The modifying of data by "functional transformation" is an approach to develop algorithms that are usually easier, faster and simpler to program than conventional algorithms (simple recursive algorithms, divide and conquer algorithms, dynamic programming algorithms, among others). By the functional transformation, a set of functions define are used to transform a set of structured data from its original form in another form of greater utility or with a specific interest. It is expected that transforming functions being "pure functions" and therefore these should be self-contained functions (i.e., data can be freely ordered and rearranged without entanglement or interdependencies) and stateless (i.e., that executing of the same function or specific set of functions on the same input will always result in the same output data) [19-21]. By FEDS, a strategy based on "non-pure" functions are used because an infrared spectrum is a data collection with a fixed order in function of vibrational energy [19].

When FEDS transform is applied, a finite approximation method is used to compute the derivatives of the spectra. Usually, derivative algorithm utilizes the signal resolution to compute differences $(\Delta v$ $=\left|v_{j}-v_{\mathrm{i}}\right|$ where $\Delta v$ is the separation between adjacent data). In the Equation 1 is showed the finite approximation of the first derivative for FTIR spectrum which is plotting usually in function of $v$ :

$$
y^{\prime}=\frac{d s}{d v} \approx \frac{\Delta s}{\Delta v}=\frac{s\left(v_{j}\right)-s\left(v_{i}\right)}{v_{j}-v_{i}}
$$

where $s$ and $\Delta s$ denote the signal for specific values of $v$ and the difference between adjacent signals, respectively. For another spectrum usually used in analytical sciences, the ultraviolet-visible spectrum, the plotting of data is typically described as a function of $\lambda[12]$.

The working tool in the FEDS transform is called Function P and it can be understood as a functional transformation that contracts the signals of FTIR spectrum in a function of critical points without to change the relative position of them.

The steps associated with the calculation of Function $P$ are:

The derivative first of the inverse of normalized spectrum is easily calculated from spectrum of absorbance $\left(a_{j}\right)$ as a function of $v$ :

$$
\frac{d a_{N, j}{ }^{-1}}{d v} \approx \frac{\Delta a_{N, j}{ }^{-1}}{\Delta v}=\left(\frac{1}{v_{j}-v_{j-1}}\right)\left[\left(\frac{1}{a_{N, j}}\right)-\left(\frac{1}{a_{N, j-1}}\right)\right]
$$

Assuming that $v_{j}-v_{i}$ is always a constant (this assumption is valid for almost all instrumental equipment), Equation 12 is rewritten to be

$$
p=\left(\frac{1}{a_{N, j}}\right)-\left(\frac{1}{a_{N, j-1}}\right)
$$

where $p$ denotes an auxiliary function $p$ in order to simplify the notation. Since Equation 13 defines positive and negative values, $|p|$ is calculated and the signals are amplified by the calculation of corresponding square root (note that absolute value operator is congruent with the definition given by equation 1, i.e., $p$ is a segment associated with fluctuation of SLF between two consecutive wavenumbers). In this point, values of absorbance have been distorted by the transformation, however, by conditional functions the values of absorbance can be recovered exactly in the wavenumber with greater significance. Finally, Function P, here defined to be FEDS intensity $\left(b_{w}\right)$ in order to avoid confusion with perturbation function, is defined to be

$$
b_{w}=\left(1+a_{N}\right) \frac{1}{\sqrt{|p|}}
$$


where $\left(1+a_{N}\right)$ is only an amplification factor for the assignation of a weight congruent with absorbance intensity. Note that, strictly speaking, equations have not limitations related with the technique, in consequence, equations should be useful for the analysis of any spectra or spectrum-like function. FEDS has been used for quantification of water contents in mixture with organic acids, determination by spectral analysis the dimerization constant and deconvolution of mid-infrared spectrum of quaternized poly(vinyl chloride) for assignation of signals [12, 22].

\section{Methodology}

The study of end-spectrum noise and its connecting with FEDS transform were performed in two stages: (i) a theoretical study using simulated spectra with basic SLF (straight-line spectrum and Gausstype spectrum) and (ii) the study of HCMS, in specific, bacterial biolayers of $E$. coli.

\subsection{Theoretical study by basic spectrum line functions}

\subsubsection{Analysis of SLF with constant noise}

Two types of straight-line spectra were analyzed: $b_{w}=k_{1}$ where $k_{1}$ is a constant and $b_{w}=k_{1} w+k_{2}$ where both $k_{1}$ and $k_{2}$ are constants. Simulation of straight-line spectra were performed for several synthetic noise $(r= \pm 2.0, \pm 4.0$ and \pm 6.0$)$, values of $k_{1}=4.0$ and $k_{2}$ $=1.0$, and for two range of data (1-50 and 1-30). Later, $r$ and SNR were calculated by linear correlation fit of data and by the use of equations 10 and 11 .

\subsubsection{Analysis of SLF with variable noise}

In order to analyze the capacity to $r$ to describe a non-constant noise in the straight-line spectrum a perturbation function $(P)$ was be used. $P$ is the function of a standing wave given by

$$
P=\frac{1}{a_{w p}} \sin \left(\frac{2 \pi w}{\lambda_{w p}}-t_{w p}\right)
$$

where $1 / a_{w p}, \lambda_{w p}$ and $t_{w p}$ are the amplitude, wavelength and displacement, respectively. Thus, $P$ permits the modeling of controlled oscillatory noise which can be included in the spectrum by a simple sum of functions. Perturbation function was applied to straight-line spectra Gauss-type spectra. In the first case, for straight-line spectrum with $k_{1}=3.0$ and $k_{2}=0.5$, a standing wave defined by $a_{w p}=2.5, \lambda_{w p}=0.2 \pi^{2}$ and $t_{w p}=2.5$ was used to produce a known-synthetic noise.

On the other hand, Gauss-type spectrum is a term used to indicate that the spectrum signal or a consecutive signal collective that are being analyzed can be described by a Gaussian function $\left(G_{0}\right)$. For theoretical analysis of noise and SNR, a Gauss-type analysis window can be drawn using a Gaussian function with a center $\left(w_{0}\right)$ and standard deviation $(s)$ defined to be $w_{\min }<w<w_{\max }$. Thus, $G_{0}$ is given by

$$
G_{0}=\frac{1}{s \sqrt{2 \pi}} \exp \left(\frac{-\left(w-w_{0}\right)^{2}}{2 s^{2}}\right)
$$

$G_{0}$ was defined for $w_{0}=600, s=5$ and $10, \Delta w=1,2,3,4$ and 5 for several analysis windows and $w_{0}=650, s=10, \Delta w=1$ and 2 for several analysis windows. In this case, perturbation function was defined by $a_{w p}=25, \lambda_{w p}=0.2 \pi^{2}$ and $t_{w p}=3.4$ and added to $G_{0}$ with $w_{0}=650$ and $s=30$.

\subsection{Study of $E$. coli biolayers}

\subsubsection{Preparation of samples and recording of spectra}

In order to exemplify the applicability of analysis of end-spectrum noise and its connecting with FEDS transform in a real system, a bacterial biolayer was deposited on ultrafiltration cellulose membrane; membrane was previously modified using a spectral marker (CISM ${ }^{\circledR}$, Mindtech s.a.s., Colombia) which produces a characteristic mid-infrared signal at $2268 \mathrm{~cm}^{-1}$ which is absent in the bacterial spectra and, in consequence, the presence of that signal can be directly associated with the cellulose support. In this study, this signal was used to produce a signal with a Gaussian-like shape. As bacterial model was used E. coli supplied by bank of bacterial strains of Research Group in Science with Technological Applications (Universidad del Valle, Colombia). Later, biolayer were dried at $40{ }^{\circ} \mathrm{C}$ per 12 hours. Finally, biolayer were analyzed using an infrared spectrophotometer with attenuated transmission reflectance with a ZnSe crystal (FTIR-ATR, IR-Affinity, Shimadzu Co). Biolayer were performed in triplicate, and average spectra for each replicate were recorded by 20 scans from 500 to $4000 \mathrm{~cm}^{-1}$.

Data were extracted in file format txt in order to carry out the analysis using a spreadsheet. Two analysis windows were selected to exemplify straight-line spectra: $3700-4000 \mathrm{~cm}^{-1}$ with $k_{1}=0$ and $2500-2700 \mathrm{~cm}^{-1}$ with $k_{1} \neq 0$, and one analysis window with Gausstype spectrum $\left(2200-2400 \mathrm{~cm}^{-1}\right)$.

\subsubsection{Pre-treatment of data}

For each analysis window, data were normalized respect to the values of minimum and maximum absorbances $\left(a_{j}-a_{\min }\right.$, respectively).

$$
b_{j}=\frac{a_{j}-a_{\min }}{a_{\max }-a_{\min }}
$$

In order to avoid calculation mistakes resulting to scaling from 0 to 1 , the zero absorbance was approximated by the calculation of average value between two adjacent values of absorbance satisfying that $b_{j-1}<b_{j}<b_{j+1}$ where $b_{j}=0$.

Since derivative spectrum is strongly sensitive to noise in the original signal, the smoothing of noise was decreased by the use of average-based spectral filter (ABSF) [12]. ABSF is given by

$$
\operatorname{ABSF}\left(b_{i} ; N=20\right)=\left.\frac{1}{3} \sum_{j}^{j+2}\left(b_{j}\right)\right|_{N=1} ^{N=20}
$$

ABSF is the moving average with a data window of 3 and 20 cycles $(N=20)$. As function is modified by the use of Equation 18, the 
same transformation of data is performed on function domain in order to correct small displacements respect to original spectrum (i.e., maximum points in original spectrum should be the same in the Function $\mathrm{P}$ ).

\section{Results and discussion}

\subsection{Analysis of different types of SLF}

\subsubsection{Straight-line spectrum}

Straight-line spectrum is the simplest situation that can be imagined, however, in this case, it is possible to identify different situations where the line shape in the analysis window could be like a straightline; typical examples are spectrum segments in very narrow or sharp bands of FTIR spectrum. A first case is identified when the SLF is described by $b_{w}=k_{1}$ where $k_{1}$ is a constant and $r=0$. But also, SLF can be described by $b_{\mathrm{w}}=k_{1} w+k_{2}$ or $b_{\mathrm{w}}=-k_{1} w+k_{2}$ where both $k_{1}$ and $k_{2}$ are constants and therefore $r=k_{1} / 2$.

Therefore, for linear SLF there is an intrinsic noise or "geometric noise" $\left(r_{0}\right)$ of geometrical nature associated with the slope and that can be used to characterize the noise in function of $w\left(r_{w}\right)$. Because $r_{0}$ is an absolute minimum, normalized spectrum can be used to define a maximum value of $S N R$ given that $S N R$ is maximum when $r$ achieves its minimum value, and $S N R$ achieves its minimum value when $b_{w}=r_{0}$, i.e.,

$$
1<S N R \leq\left(1 / r_{0}\right)
$$

because $r>r_{0}$, "SNR scale" for the description of end-spectrum noise as a function of $w$ can be defined; this scale is seen to be useful for monitoring of noise effect along the spectrum and the noise changes produced by smoothing techniques. Equation 19 can be rewritten as $S N R_{1}<S N R \leq S N R_{0}$ being $S N R_{1}=b_{w} / r_{w}$ with $b_{w}=r_{w}$, $S N R_{0}=1 / r_{0}$ and in the interval $S N R=b_{w} / r_{w}$.

In the table 1 the results of the simulation of straight-line spectrum with constant noise is shown. From input parameters used in the simulation, by the slope of ideal straight-line spectrum and equation 10 is concluded that $r_{0}=2.0(0.0100)$ and $S N R_{0}=100.5$ for range " $a$ " whereas $r_{0}=2.0(0.0165)$ and $S N R_{0}=60.5$ for range " $b$ " (in Table 1 , the value in parentheses adjacent to $r_{0}$ corresponds to the value of $r_{0}$ from the normalized data and $a$ and $b$ denote the number of data in the corresponding analysis windows). It can be seen that for the same range of data, $\Delta w$ and different amounts of synthetic noise, $r_{0}$ can be calculated from linear correlation fit of $n-1$ data whereas $r$ and SNR are easily calculated by equations 1 and 10; however, it is clear that linear correlation fit cannot be used to determine the noise amount in the spectrum.

On the other hand, from simulation shown in Table 1 can be seen that $r$ is an adequate descriptor for the amount noise in the spectrum. Note that, if $r$ is increased then $S N R$ is decreased according to the expected behavior. In addition, the value of $n$, that is directly related with $\Delta w$ and the range of data, is seen to produce a marked effect on values of $r$ that are calculated from normalized data and on the corresponding values of $S N R$; the above can be understood by the relation among $S N R$ and $r$, and by the dependence of $r$ of the following parameters: $k_{1}, w_{\max }, w_{\min }$ and $n$. Note that, for a SLF described by a straight-line function, the change in two consecutive values of absorbance is $\Delta b=b_{w+1}-b_{w}$ and the corresponding slope is $k_{1}=\Delta b / \Delta w$. According to equation $10, r=\Delta b / 2$ and therefore $r=$ $k_{1} \Delta w / 2$. By the use of equation 2 easily can be concluded that

$$
r=\frac{k_{1}\left(w_{\max }-w_{\min }\right)}{2 n}
$$

But also, it can be seen in Table 1 that $R / R_{n}$ is not affected by $n, \Delta w$ or the range of data suggesting that this ratio can be used for the comparison of different SLFs. In addition, values $k_{2}$ is seen to has not any effect in the description of $r$ being only important $k_{1}$ as consequence of the previously deducted conclusion: $r_{0}=k_{1} / 2$. In the previous analysis, the correct description of noise by $r$ can be explained since synthetic noise was constant.

It is seen from Table 2 that $r_{0}$ can be predicted by a linear correlation fit and therefore this procedure can be used not only to estimate the valor of $r_{0}$ but also for the making of the noise scale using the equation 19. In addition, if $k_{1}$ is increased for the same function $P$, the synthetic noise is decreased respect to geometric noise, i.e., the contribution of added noise using the function $P$ is decreased; the above behavior is satisfactorily described by $R / R_{n}$ since this ratio changes from 0.13 to 0.99 .

In Figure $1 \mathrm{~A}$ the function $P$ used has a node denoted $q$ ( $q$ indicates

Table 1. SLF simulation of straight-line spectrum with constant noise $b_{n}(r= \pm 2.0, \pm 4.0$ and \pm 6.0$)$ for ideal straight-line spectrum described by $b_{w}=k_{1} w+k_{2}\left(k_{1}=4.0\right.$

\begin{tabular}{|c|c|c|c|c|c|c|}
\hline$n$ & $b_{n}$ & $r_{n}$ & $S N R_{n}$ & $r$ & SNR & $R / R_{n}$ \\
\hline \multirow[t]{3}{*}{50 (a) } & \pm 2.0 & $2.0(0.010)$ & 99.5 & $2.0(0.010)$ & 99.5 & 1.00 \\
\hline & \pm 4.0 & $2.0(0.010)$ & 100.5 & $4.0(0.020)$ & 50.3 & 0.50 \\
\hline & \pm 6.0 & $2.0(0.010)$ & 101.5 & $6.0(0.030)$ & 33.8 & 0.33 \\
\hline \multirow[t]{3}{*}{30 (b) } & \pm 2.0 & $2.0(0.017)$ & 59.5 & $2.0(0.017)$ & 59.5 & 1.00 \\
\hline & \pm 4.0 & $2.0(0.017)$ & 60.5 & $4.0(0.033)$ & 30.3 & 0.50 \\
\hline & \pm 6.0 & $2.0(0.016)$ & 61.5 & $6.0(0.049)$ & 20.5 & 0.33 \\
\hline
\end{tabular}
and $\left.k_{2}=1.0\right)$, range of data 1-50 (a) and 1-30 (b) with $\Delta w=1\left(b_{n}=b_{w} \pm r\right)$.

"For simulation, $r_{0}=2.0(0.010)$ and $S N R_{0}=100.5$ for range " $a$ " and $r_{0}=2.0(0.017)$ and $S N R_{0}=60.5$ for range " $b$ ". In parenthesis are shown the values of $r_{n}$ and $r$ calculated from normalized spectra. In addition, $r_{n}$ and $S N R_{n}$ are the values of $r$ and $S N R$ calculated by linear correlation fit of data in that order, $r_{w}$ and $S N R$ are values calculated from equations 10 and 11 and $R / R_{n}=S N R / S N R_{n}$ 
Table 2. SLF simulation of straight-line spectrum described by $b_{w}=k_{1} w+k_{2}\left(k_{1}=1.0\right.$ to 16.0 and $\left.k_{2}=1.0\right)$, range of data $1-30$ and $\Delta w=1$.

\begin{tabular}{ccccccc}
\hline$k_{1}$ & $r_{0}$ & $r_{n}$ & $S N R_{n}$ & $r$ & $S N R$ & $R / R_{n}$ \\
\hline 1 & $0.5(0.0161)$ & $0.47(0.0130)$ & 76.8 & $3.67(0.1005)$ & 9.9 & 0.13 \\
2 & $1.0(0.0164)$ & $0.97(0.0160)$ & 67.2 & $3.72(0.0567)$ & 17.6 & 0.26 \\
4 & $2.0(0.0165)$ & $1.97(0.0149)$ & 62.5 & $3.91(0.0317)$ & 31.5 & 0.50 \\
8 & $4.0(0.0166)$ & $3.97(0.0166)$ & 60.2 & $4.72(0.0197)$ & 50.7 & 0.84 \\
12 & $6.0(0.0166)$ & $5.97(0.0168)$ & 59.5 & $6.09(0.0171)$ & 58.3 & 0.98 \\
16 & $8.0(0.0166)$ & $7.97(0.0168)$ & 59.4 & $8.00(0.0169)$ & 59.2 & 0.99 \\
\hline
\end{tabular}

* Synthetic noise was added to $b_{w}$ using a standing wave with the following parameters: $a_{w p}=0.08,\left(2 \pi / \lambda_{w p}\right)=1 /(0.1 \pi)$ and $t_{w p}=3.8$. (in parenthesis are shown the values of $r_{0}, r_{n}$ and $r$ calculated from normalized spectra).
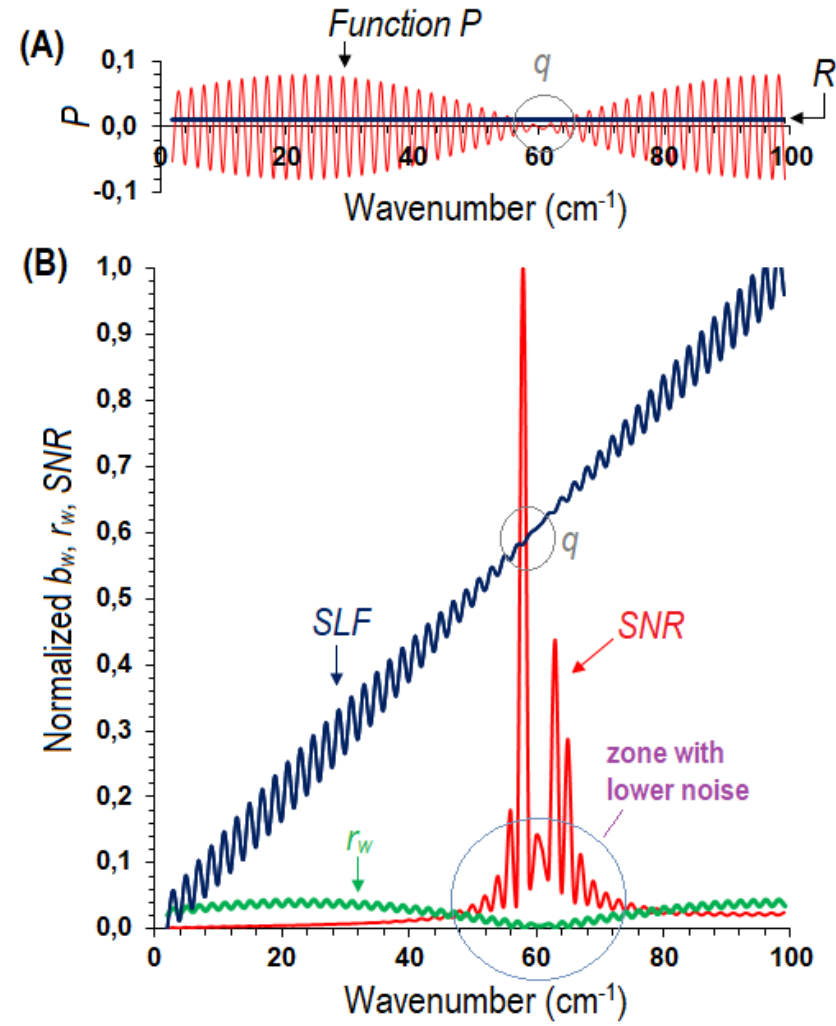
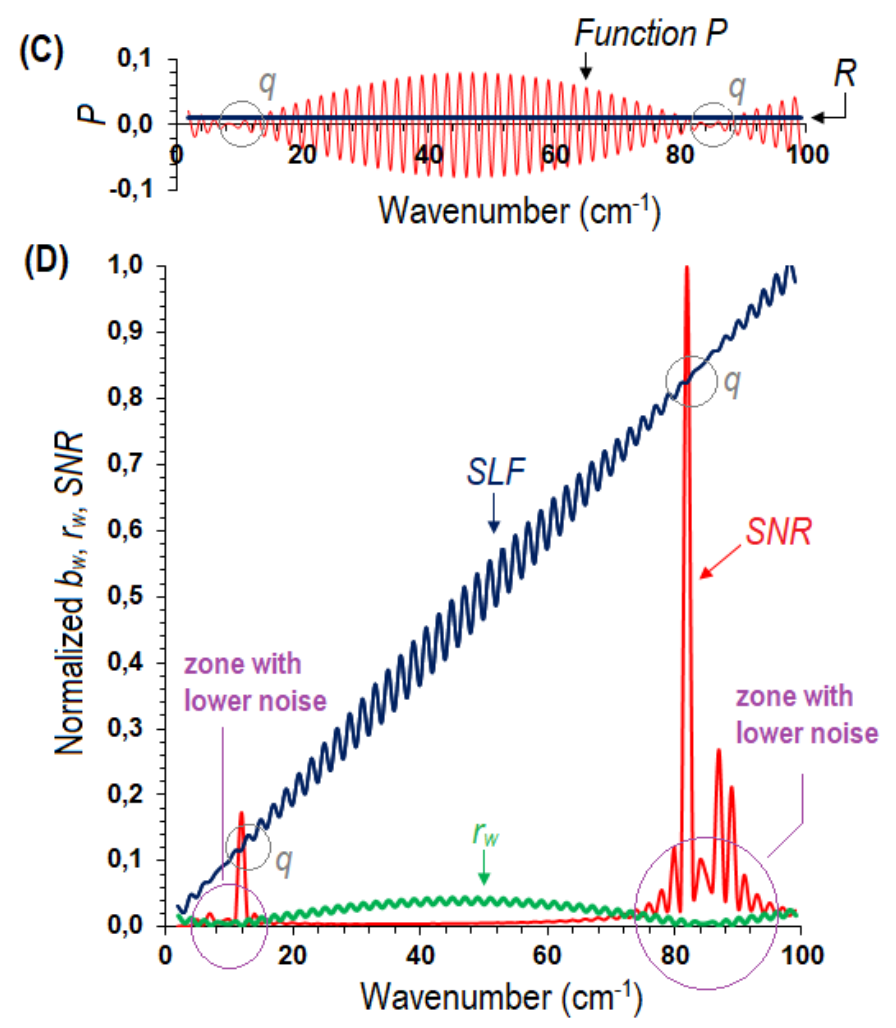

Figure 1. (A) Function $P$ for a standing wave defined by $a_{w p}=2.5, \lambda_{w p}=0.2 \pi^{2}$ and $t_{w p}=2.5$, (B) SLF resulting to add the function $P$ to the straight-line spectrum given by $k_{1}=3$ and $k_{2}=0.5$ with corresponding $r_{w}$ and $S N R$, (C) Function $P$ for a standing wave defined by $a_{w p}=2.5, \lambda_{w p}=0.2 \pi^{2}$ and $t_{w p}=3.5$, and (D) SLF, $r_{w}$ and $S N R$ resulting to add the function $P$ to the straight-line spectrum $\left(k_{1}=3\right.$ and $\left.k_{2}=0.5\right)$ (scales have been normalized respect to maximum value to ease the comparison).

the zone with lower noise); thus, when the addition of synthetic noise to straight-line function is performed, the new function that is obtained is shown in Figure 1B. It can be seen that $q$-point corresponds to node in the SLF, but also, it can be seen that minimum value of $r_{w}$ is corresponded with this point and with the zone of lower noise in the SNR function. Similar results are obtained when synthetic noise is placed at the ends of analysis window (see Figures $1 \mathrm{C}$ and 1D).

The above demonstrate that is possible the use of $r$ for to detect signals with less noise and use them to amplify the signals by multiplication of original spectrum and SNR-based spectrum, in addition, it's possible to perform a spectrum transformation by the use of SNR in function of $w$ in order to improve the analysis of information contained in the analysis window (exemplification of previous ideas are shown for the analysis of FTIR spectrum).

For an analysis window with shape of curved-line, the segment described by two consecutive values can be assumed to be a straight-line because typically $\Delta w$ is small and, in consequence, $r$ can be easily calculated. In addition, $r_{0}$ can be determined by correlation fit, drawing the corresponding function and applying the Eq. (1). However, in many cases, the analysis of complete band and not only a segment can be useful. Thus, the study of SLF with more complex shapes (Gaussian shape) is analyzed below. 
Table 3. Values of geometric noise $\left(r_{0}\right)$ and maximum SNR for normalized Gaussian-type spectrum as a function of $w 0, s$ and $\Delta w$. Corresponding estimations by equation 15 for $r_{0}$ and $S N R$ are shown as $r_{x}$ and $S N R x$, respectively.

\begin{tabular}{|c|c|c|c|c|c|c|c|c|c|}
\hline wo & $s$ & $\Delta w$ & Range & $n$ & $r_{0}$ & SNR & $r_{x}$ & SNRx & row \\
\hline \multirow[t]{7}{*}{600} & 5 & 5 & $585-620$ & 35 & 0.12496 & 8.00 & 0.1250 & 8.0 & (a) \\
\hline & & 4 & 584-616 & 32 & 0.1108 & 9.03 & 0.1111 & 9.0 & (b) \\
\hline & & 3 & $584-620$ & 36 & 0.0769 & 13.01 & 0.0769 & 13.0 & (c) \\
\hline & & 2 & $582-618$ & 36 & 0.0526 & 19.02 & 0.0526 & 19.0 & (d) \\
\hline & & 2 & $560-640$ & 80 & 0.0244 & 41.00 & 0.0244 & 41.0 & (e) \\
\hline & & 2 & $550-650$ & 100 & 0.0196 & 51.00 & 0.0196 & 51.0 & (f) \\
\hline & & 1 & $582-623$ & 41 & 0.0238 & 42.02 & 0.0238 & 42.0 & (g) \\
\hline \multirow[t]{2}{*}{600} & 10 & 2 & $550-650$ & 100 & 0.0196 & 51.00 & 0.0196 & 51.0 & (h) \\
\hline & & 1 & $550-650$ & 100 & 0.0099 & 101.00 & 0.0099 & 101.0 & (i) \\
\hline \multirow[t]{2}{*}{650} & 10 & 2 & $600-700$ & 100 & 0,0196 & 51,00 & 0.0196 & 51.0 & (j) \\
\hline & & 1 & $600-700$ & 100 & 0,0099 & 101,00 & 0.0099 & 101.0 & (k) \\
\hline
\end{tabular}

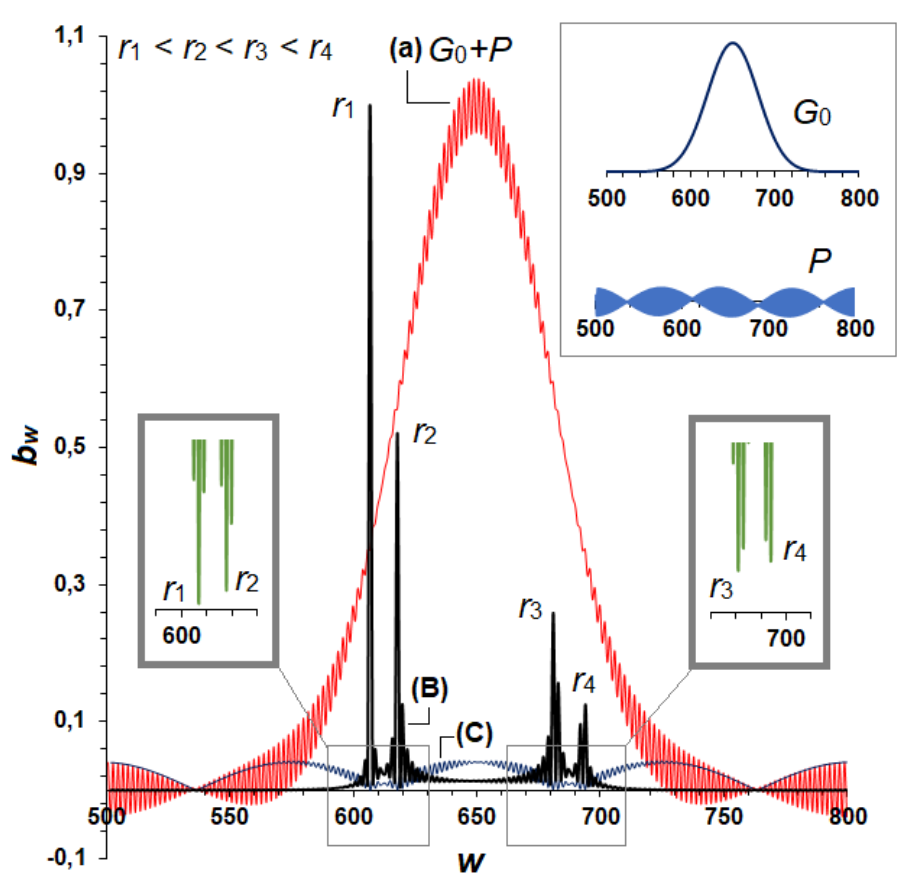

Figure 2. (a) Gauss-type function with variable synthetic noise defined by a function $P\left(a_{w p}=25, \lambda_{w p}=0.2 \pi^{2}\right.$ and $\left.t_{w p}=3.4\right)$ added to $G_{0}\left(w_{0}=650\right.$ and $\left.s=30\right)$, (b) $r$ as a function of $w$ for $G_{0}+P$, and (c) $S N R$ as a function of $w$. In addition, the magnification of SNR to show the minimum values of $r$ and the correct correlation with the respective maximum values of $S N R$ (left and right boxes indicating $r_{1}, r_{2}, r_{3}$ and $r_{4}$, respectively). In the top box the $G_{0}$ and $P$ functions are shown.

\subsubsection{Gauss-type spectrum}

A first observation is that Gauss function has a set of values of $r_{0}$ depending on the analysis window. The above is a direct conclusion from analysis of straight-line spectrum, thus, because the slope is directly related with the value of $r_{0}$, a change of slope is understood as a change into the $r_{0}$, in consequence, different values of $r_{0}$ for different ranges of the Gauss function through SLF can be determined. However, like to the observed for straight-line spectrum, for Gauss-type spectrum the value of $r_{0}$ is strongly affected by the wavenumber range (i.e., $w_{\min }$ and $w_{\max }$ ), $\Delta w$ and $n$. It can be seen in the Table 3 that for different ranges and resolutions, rows from a to $\mathbf{d}$, different values of $r_{0}$ are obtained for the same Gauss function. In addition, when resolution is a constant for similar analysis windows, by instance for rows from $\mathbf{d}$ to $\mathbf{f}$, different values of $r_{0}$ are obtained for the same function. On the other hand, it can be seen that for the same values of $\Delta w$ and $n$, the values of $r_{0}$ are the same (see rows $\mathbf{f}, \mathbf{h}$ and $\mathbf{j}$, and rows $\mathbf{i}$ and $\mathbf{k}$ ); the above illustrates the relevant of these parameters for the determination of $r_{0}$ and the careful to take during the noise behavior for different analysis windows. By the analysis of results obtained for several Gaussian functions, it can be concluded that for this type of functions an acceptable estimation of $r_{0}\left(r_{\mathrm{x}}\right)$ can be calculate by

$$
r_{x}=\frac{\Delta w}{n+\Delta w}
$$

Results of estimation of $r_{0}$ and SNR are shown in the Table 3. It can be seen that to obtain the value of $r_{0}$ in a Gauss-type spectrum only it must be known $\Delta w$ and $n$ (rows $\mathbf{f}, \mathbf{h}$ and $\mathbf{j}$ or rows $\mathbf{i}$ and $\mathbf{k}$ ). As it was previously concluded, $r$ can be calculated by equation 10 .

In Figure 2 is shown a Gauss-type function with variable synthetic noise defined by a function $P$ which was added to $G_{0}\left(P\right.$ and $G_{0}$ are shown in the top box); it can be identified clearly the distribution of noise in the resulting function. Also, it is shown the description of noise by the use of definition of $r$ as a function of $w$ for $G_{0}+P$. The description achieved by $r$ is completely congruent with $G_{0}+P$ function $\left(r_{1}<r_{2}<r_{3}<r_{4}\right)$. Finally, $S N R$ as a function of $w$ showed a correct description of noise distribution, permitting the identification of points with lower noise.

\subsubsection{Analysis of biolayers of $E$. coli}

In Figure 3 is shown the average spectrum obtained for biolayer of E. coli (only region between $2100-4000 \mathrm{~cm}^{-1}$ is shown due the three analysis windows selected to exemplify the analysis can be observed in this part of spectrum). It can be seen that spectrum shows typical signals associated with vibrations associated to hydroxyl and amino groups $\left(3000-3600 \mathrm{~cm}^{-1}\right)$, vibrations $\mathrm{C}-\mathrm{H}$ of $\mathrm{CH}_{3}$ and $\mathrm{CH}_{2}\left(2800-3000 \mathrm{~cm}^{-1}\right)$, and it is evident that overlapping of signals. By the above, the spectrum analysis is limited by direct analysis of vibrations and it is in these situations when spectrum digital transformation is useful. 

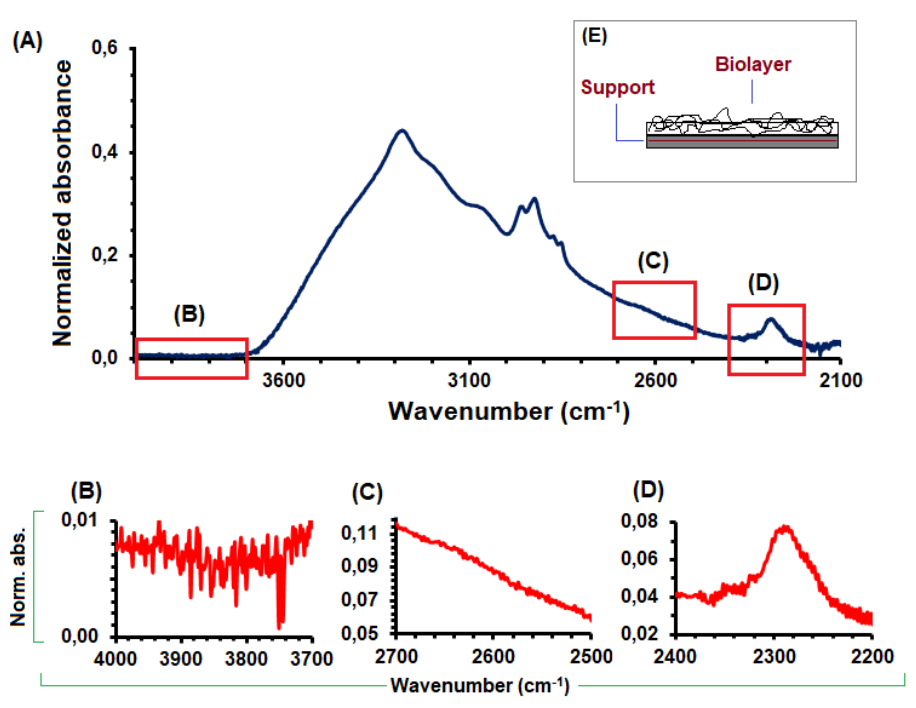

Figure 3. (A) Average IR spectrum of $E$. coli between 2100 and $4000 \mathrm{~cm}^{-1}$, (B) and (C) analysis windows 1 and 2 between $3700-4000 \mathrm{~cm}^{-1}$ and $2500-2700 \mathrm{~cm}^{-1}$, respectively, as models of straight-line spectra, (D) analysis window 3 between $2200-2400 \mathrm{~cm}^{-1}$ as model of Gauss-type spectrum and (E) illustration of bacterial biolayer.
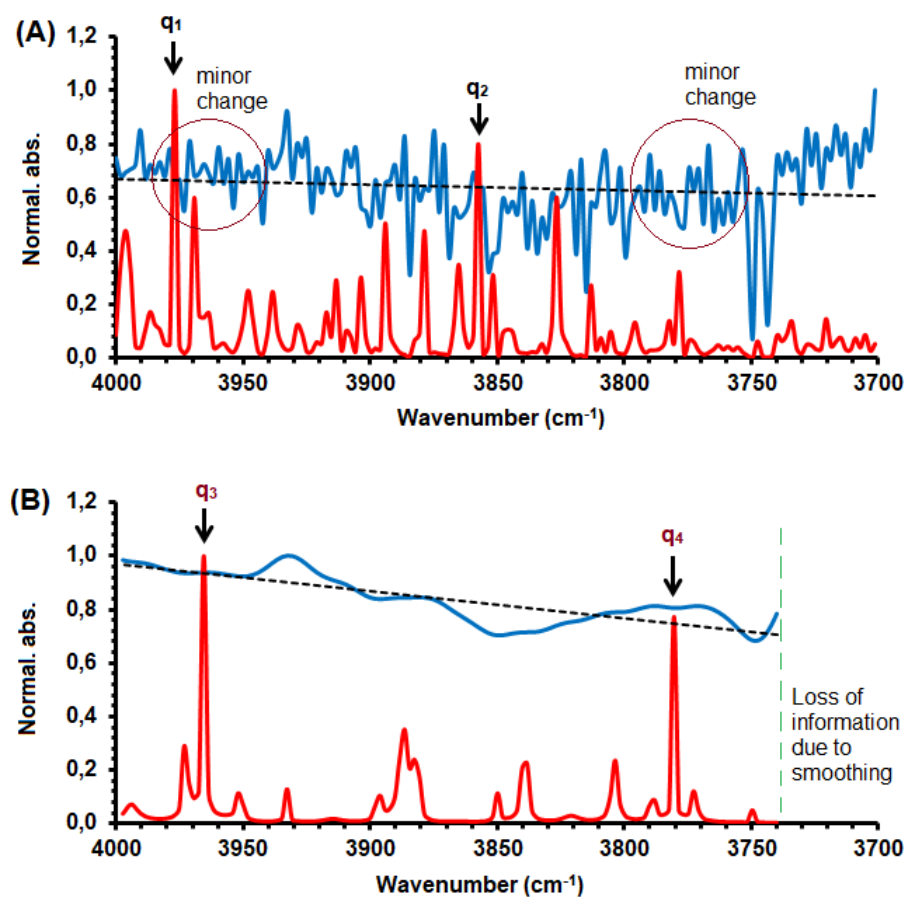

Figure 4. (A) Analysis window between $3700-400 \mathrm{~cm}^{-1}$ corresponding to normalized average spectrum ("blue plot" with $1 / 0.0037$ as normalization factor; dash line is the corresponding correlation fit) and normalized SNR-based spectrum ("red plot" with $1 / 252.2$ as normalization factor) and (B) Analysis window between $3700-400 \mathrm{~cm}^{-1}$ corresponding to normalized-smoothed average spectrum ("blue plot" with 1/0.0027 as normalization factor) and normalized SNR-based spectrum ("red plot" with 1/19436.4 as normalization factor).
In region $\mathrm{B}$, analysis window can be assumed to be a straight-line spectrum with slope zero, however, fluctuation of signal evidences the presence of residual noise in this analysis window (the maximum change is $\sim 1 \%$ of maximum signal, therefore the signal recorded in the spectrum can be considered to be noise). In region $\mathrm{C}$, analysis window can be assumed to be a straight-line spectrum with slope different to zero with a subjective overlapping due to it is not possible identify a signal (a small fluctuation which could be noise being in this point lower than $\mathrm{B}$, here, the maximum change is $\sim 0.1 \%$ of maximum signal). Finally, in the region $\mathrm{D}$, analysis window can be assumed to be a Gauss-type window with intermediate noise in comparison with $\mathrm{B}$ and $\mathrm{C}$.

The analysis of residual noise in the region B is shown in Figure 4. Thus, from Figure 4A, the $S N R$ shown that two signals excel $\left(\mathrm{q}_{1}\right.$ and $\mathrm{q}_{2}$ with SNR of 1.0 and 0.8 in the normalized spectrum with maximum $S N R=252.2$ in the original spectrum; however, in this analysis window the $a_{\max }$ is only 0.0037). If spectrum is smoothed using moving average, with a cycle of twenty and a window of three, the noise is decreased and the effect of smoothing is manifested in a simplification of SNR-based spectrum (see Figure 4B). As result of smoothing, two signals $\mathrm{q}_{3}$ and $\mathrm{q}_{4}$ are visualized and increased respect to other signals. By near spectroscopy, the vibrations associated to overtones can be identified, thus, between 3700-400 $\mathrm{cm}^{-1}$ is expected to see, for example, two second-order overtones of the $\mathrm{CH}$ deformation ( 3945 and $3757 \mathrm{~cm}^{-1}$ ). However, these signals should be weaker by 1/10-1/50 than the bands of the first-order combination and usually they are neglected [20]. In our case, these signals also are neglected because information obtained from them is not important in the mid-infrared, but in the framework of our analysis of noise, it is evidenced that potential signals could be hidden in the noise and be revealed by analysis of SLF using endspectrum noise concept.

In the region $\mathrm{C}$, analysis window is shown in Figure 5A for original spectrum (blue plot), and SNR as a function of wavenumber (red plot). Linear correlation fit is described by $a=0.0027 w-6.1553$ with correlation coefficient of 0.9906 . In consequence, $R$ and $r_{0}$ are estimated to be 0.00734 and 0.00135 , respectively. In order to minimize the noise, a smoothing is performed (with a window of 3 and 20 cycles) and smoothed spectrum can be seen in Figure 5B. Note that, 3 new signals can be identified in the SNR-based spectrum $\left(\mathrm{q}_{1}, \mathrm{q}_{2} \mathrm{q}_{3}\right.$ and $\left.\mathrm{q}_{4}\right)$. These signals correspond to spectrum data in the analysis window with low SNR; when smoothing is performed, segments where the distance between points is very small are smoothed producing a straight-like segment with slope near to zero, thus, by definition of $r$, as slope is decrease then $r$ is decreased and SNR is increased. Therefore, points observed in SNR-based spectrum are associated with larger values of SNR and could be understood to be "hidden" signals. In the figure C is shown a comparison between FEDS intensity obtained for smoothed spectrum. The evident correlation suggests that capacity of FEDS to deconvoluted the spectrum is a result of increasing of SNR, in other words, FEDS is capable of find and increase the points on SLF with greater SNR, being the reason of capacity of FEDS transformation of increase the spectral resolution of signals. 

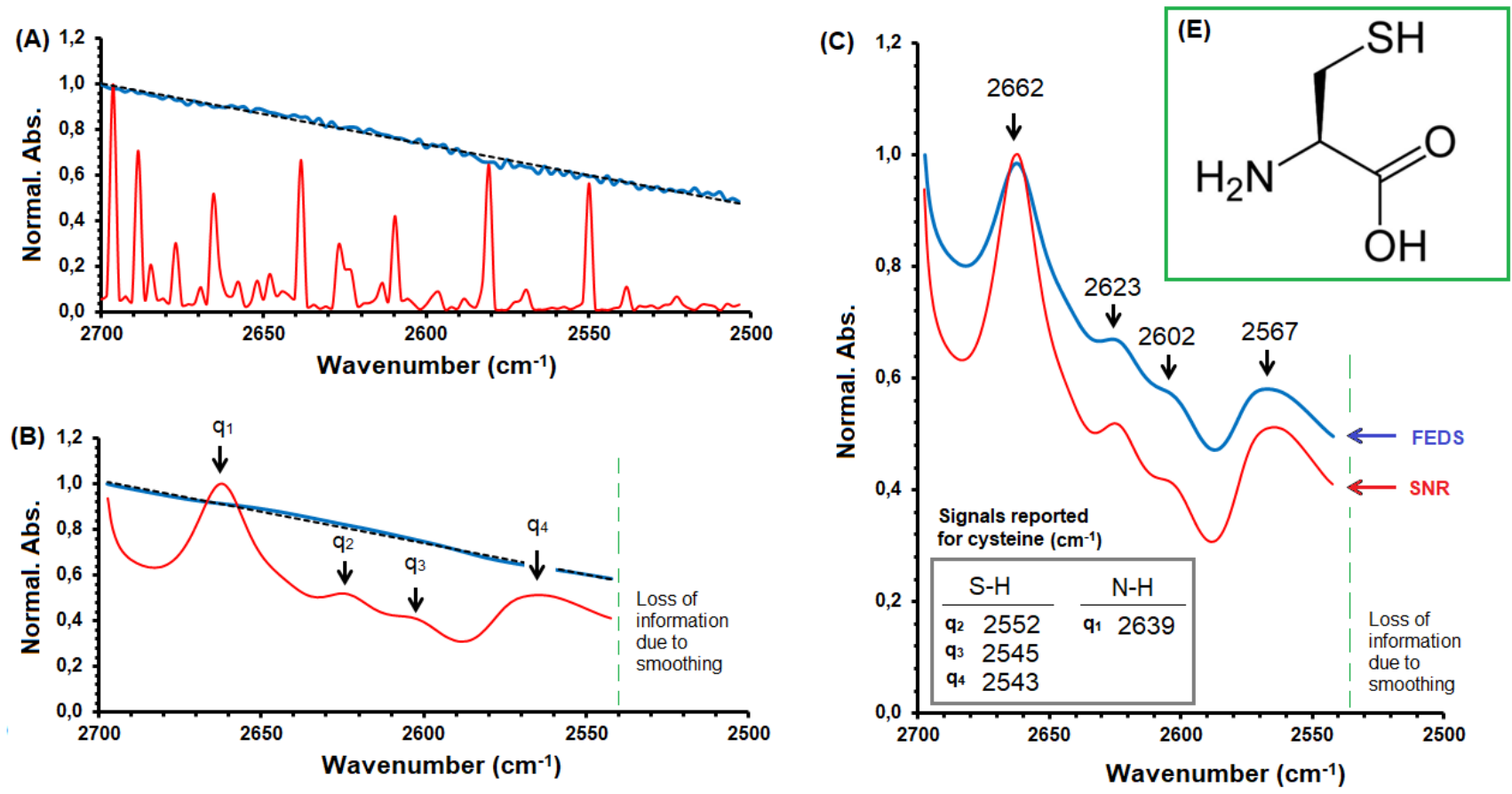

Figure 5. (A) Analysis window between $2500-2700 \mathrm{~cm}^{-1}$ corresponding to normalized average spectrum ("blue plot" with $1 / 0.0355$ as normalization factor; dash line is the corresponding correlation fit) and normalized SNR-based spectrum ("red plot" with 1/2667 as normalization factor), (B) Analysis window between $2500-2700 \mathrm{~cm}{ }^{-1}$ corresponding to normalized-smoothed average spectrum ("blue plot" with 1/0.035 as normalization factor) and normalized-smoothed SNR-based spectrum ("red plot" with $1 / 593.2$ as normalization factor) and (C) comparison normalized-smoothed SNR-based and FEDS spectra.

As biolayer are formed by bacterial biofilms, surface is formed by a mixture of substances with different structural features, however, this biofilm has been reported to be constituted by glycoproteins and peptides which act as adhesins and usually have units of cysteine (see figure 5E). Cysteine has two links whit vibrations appearing in this analysis window. In general, S-H vibration has been published to be a weak vibration between $2550-2560 \mathrm{~cm}^{-1}$ or $2550-2600 \mathrm{~cm}^{-}$ 1 [21, 23]. However, a complete assignment of the vibrational spectra of L-cysteine solid state including orthorhombic and monoclinic forms by the use of a combination of computational and experimental methods show three vibrations associated with $\mathrm{S}-\mathrm{H}$ bond $\left(2543,2545\right.$ and $\left.2552 \mathrm{~cm}^{-1}\right)$ and two vibrations associated with $\mathrm{N}-\mathrm{H}$ bond (one in the analysis window at $\sim 2639 \mathrm{~cm}^{-1}$ ) [24]. The analysis permits conclude that effectivity of SNR-based spectrum and FEDS transform to improve the resolution of spectra is improved by the use of smoothing techniques, but also, the use of smoothing minimize the appearing of artefacts in the modified spectrum.

The equivalence between SNR-based spectrum and FEDS transform can be understood by the incorporation of end-spectrum noise defined in this paper. It can be seen that FEDS transform can be easily written as a function of SNR by the use of equation 10, thus

$$
b=f \sqrt{S N R}
$$

By graphical analysis can be seen that $f=\left(1+a_{n}\right)\left(a_{n-1} / 2\right)^{0.5} \approx a$.
In the region D, analysis windows for original, SNR-based and FEDS spectra (blue, red and black plots, respectively) are shown in Figure 6A. It can be seen that SNR-based and FEDS spectra are very sensitive to end-spectrum noise since both transformations are related with the changes in the SLF. Again, it is observed that smoothing improves considerably the quality of analysis (see Figure 6B).

By SNR-based and FEDS spectra four signals are identified from one single signal identified from original spectrum in the analysis window $\left(\mathrm{q}_{0}, \mathrm{q}_{1}, \mathrm{q}_{2}\right.$ and $\left.\mathrm{q}_{3}\right)$ and two minor signals with intensity lower than 0.2 (20\% compared with maximum value $\mathrm{q}_{0}$ ) and are associated with overlapping of adjacent signals, therefore, these were named overlapping' signals (see Figure 6D). On the other hand, $\mathrm{q}_{0}$ and $\mathrm{q}_{1}$ are main signals because they have the greater $S N R$. However, when SLF has slope $\sim 0$ for minimum and maximum points, being the maximum points associated with signals whereas minimum points are associated with intersection of overlapped signals completely defined (see Figure 6C), in consequence, $\mathrm{q}_{1}$ is discarded.

By the above, for the biolayer analyzed $\mathrm{q}_{0}$ at $2290 \mathrm{~cm}^{-1}$ is associated with spectral marker (-NCO) [21], $\mathrm{q}_{2}$ and $\mathrm{q}_{3}$ at 2382 and $2340 \mathrm{~cm}^{-1}$ are associated with antisymmetric $\mathrm{C}=\mathrm{O}$ stretching vibration of $\mathrm{CO}_{2}$ (g) and $\mathrm{CO}_{2}(\mathrm{aq})$, respectively; which can be produced by metabolism of biofilm and re-absorbed as result of hydrogel-type behavior [25-26]. But also, $\mathrm{q}_{3}$ is suggested that could be associated with $-\mathrm{OH}$ in $\mathrm{O}=\mathrm{P}-\mathrm{OH}$ [27-28], which is present on structure of phospholipids constituting the external surface of bacteria [29]. 

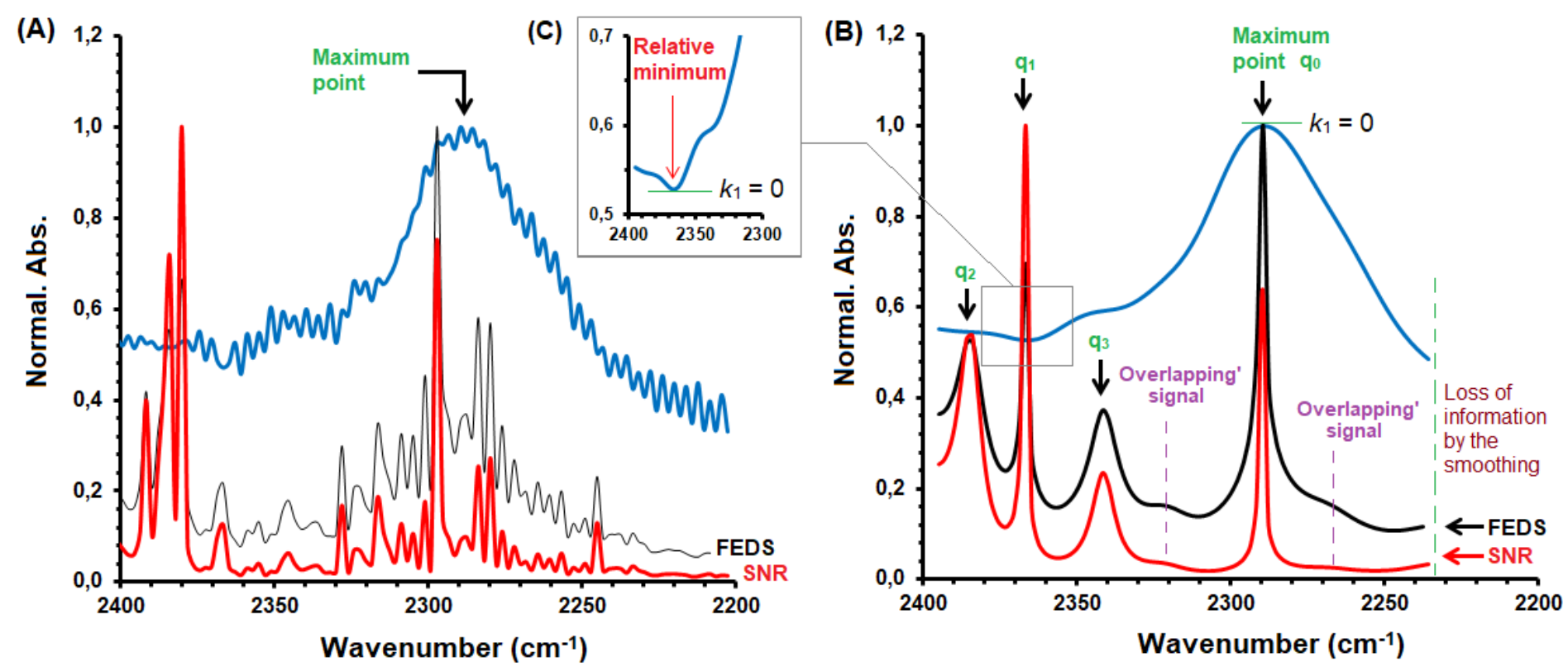

Figure 6. (A) Analysis window between $2200-2400 \mathrm{~cm}^{-1}$ corresponding to normalized average spectrum ("blue plot" with $1 / 0.026$ as normalization factor), and FEDS transformation of normalized spectrum (1/30.77 as normalization factor) and (B) Analysis window between $2200-2400 \mathrm{~cm}^{-1}$ corresponding to normalized-smoothed average spectrum ("blue plot" with 1/0.025 as normalization factor), normalized-smoothed SNR-based spectrum ("red plot" with 1/2914 as normalization factor) and FEDS spectrum ("black plot" with 1/61 as normalization factor) and (C) enlargement of spectrum segment associated with point q1.

\section{Conclusions}

It is possible to carry out the study of residual noise and the corresponding SNR in the spectrum by concept of end-spectrum noise and analysis of SLF. Thus, by SNR is possible detect and differentiate the signals with greater contribution of fluctuation of SLF associated with the noise. However, the transformation of FTIR spectrum to SNR-spectrum or FEDS-spectrum is sensitive to noise and, in consequence, smoothing should be performed in order to 茫

Conflict interest.

Authors declare that there is no conflict of interest regarding the publication of this paper.

Acknowledgements. M. Palencia thanks to University del Valle and Mindtech s.a.s. (AFICAT MT-UV 2018-2023).

\section{References}

1. E. Voigtman, nal. Chem. 69 (1997) 226-234. https://pubs.acs.org/doi/10.1021/ac960675d

2. B. Wang, A. Goodpaster, M. Kennedy, Chemom. Intellig. Lab. Syst. 128 (2013) 9-16. https://doi.org/10.1016/j.chemolab.2013.07.007

3. A.M. Sila, K.D. Shepherd, G.P. Pokhariyal, Chemom. Intell. Lab. Syst. $153 \quad(2016)$ 92-105. https://doi.org/10.1016/j.chemolab.2016.02.013

4. M. Prakash, J. K. Sarin, L. Rieppo, I. O. Afara, J. Töyräs, Chemom. Intell. Lab. Syst. 182 (2018) 166-171. https://doi.org/10.1016/j.chemolab.2018.09.010

5. B.C. Smith, Fundamentals of Fourier transform infrared spectroscopy. CRC Press - Taylor Francis Group: Boca Raton, USA. 2011.

6. M. Howard, P. Griffiths, Appl. Spec., 56 (2002) 633-639. https://doi.org/10.1366/0003702021955196 
7. B.H. Stuart. Infrared spectroscopy: fundaments and applications. Wiley: England, 2004.

8. T. Vazhnova, D.B. Lukyanov. Anal. Chem. 85 (2013) 11291-11296. https://pubs.acs.org/doi/10.1021/ac4020337

9. V. Lórenz-Fonfría, E. Padrós, Appl. Spec. 63 (2009) 791-799. https://doi.org/10.1366/000370209788701161

10. B. De Aragão, Y. Messaddeq, J. Braz. Chem. Soc., 19 (2008)1582-1594. http://dx.doi.org/10.1590/S0103-50532008000800019

11. R. Gautam, S. Vanga, F. Ariese, S. Umapathy, EPJ Tech. Instrum., 8 (2015) 2-38. https://doi.org/10.1140/epjti/s40485-015-0018-6

12. M. Palencia, J. Adv. Res. 14 (2018) 53-62. https://doi.org/10.1016/j.jare.2018.05.009

13. C. Cui, T. Fearn, Chemom. Intell. Lab. Syst. 182 (2018) 9-20. https://doi.org/10.1016/j.chemolab.2018.07.008

14. J.H. Perkins, E.J. Hasenoehrl, P.R. Griffiths, Chemom. Intell. Lab. Syst. 15 (1992) 75-86. https://doi.org/10.1016/0169$\underline{7439(92) 80028-3}$

15. K. Norris. A closer look at noise. NIR news. 3 (1992) 4-6. https://doi.org/10.1255/nirn.122

16. M. Kokalj, M. Rihtarič, S. Kreft, Chemom. Intell. Lab. Syst. 108 (2011) 154-161. https://doi.org/10.1016/j.chemolab.2011.07.001

17. S.J. Baek, A. Park, J. Kim, A. Shen, J. Hu, Chemom. Intell. Lab. Syst. 98 (2009) 24-30. https://doi.org/10.1016/j.chemolab.2009.04.007

18. A.X. Zhao, X.J. Tang, Z.H. Zhang, J.H. Liu, 9th IEEE Conference on Industrial Electronics and Applications, 2014; https://doi.org/10.1109/ICIEA.2014.6931218.

19. G. Bazar, Z. Kovacs, R. Tsenkova, PLOS One, 5 (2016) 1-15. https://doi.org/10.1371/journal.pone.0146249

20. R. Iwamoto, A. Nara, T. Matsuda, Appl. Spectr. 60 (2006) 450-458. https://doi.org/10.1366/000370206776593627

21. J. Coates, Interpretation of Infrared Spectra, A Practical Approach. In: R.A. Meyers (Ed.) Encyclopedia of Analytical Chemistry: Applications, theory and instrumentation, John Wiley \& Sons, (2006) 10815-10837. https://doi.org/10.1002/9780470027318.a5606

22. M. Palencia, T.A. Lerma, N. Afanasjeva, Eur. Polym. J., 115 (2019) 212-220. https://doi.org/10.1016/j.eurpolymj.2019.03.041

23. A. Barth, Progr. Biophys. Molec. Biol., 74 (2000) 141-173. https://doi.org/10.1016/S0079-6107(00)00021-3

24. S. Parker, Chem. Phys., 424 (2013) 75-79. https://doi.org/10.1016/j.chemphys.2013.04.020

25. X.O. Kroukamp, G. Wolfaardt, Appl. Environ. Microbiol., 75 (2009) 4391-4397. https://doi.org/10.1128/AEM.01567-08

26. T. Schadle, B. Pejcic, B. Mizaikoff, Anal. Methods (2016) 1-4. https://doi.org/10.1039/C5AY02744F

27. D. Lin-Vien, N.B. Colthup, W.G. Fateley, J.G. Grasselli. The Handbook of Infrared and Raman Characteristic Frequencies of Organic Molecule, Elsevier, Sn Diego, 1991.

28. S. Rungrodnimitchai. The Scientif. World J. (2014) 1-9. http://dx.doi.org/10.1155/2014/634837

29. N. Malanovic, K. Lohner, Biochim. Biophys. Acta (BBA) - Biomembr. $1858 \quad(2016) 936-946$. https://doi.org/10.1016/j.bbamem.2015.11.004

米

(C) MT-Pallantia Publisher (2020) 